Artículo Original

\title{
Perfil demográfico y clínico-funcional de pacientes con atrofia muscular espinal atendidos en el Instituto Teletón Santiago
}

ANDREA SOLERVICENS $\mathrm{P}^{1}$, CONSTANZA MONTENEGRO S ${ }^{2}$.

'Residente Fisiatría, Universidad del Desarrollo. 2Departamento de Fisiatría, Instituto Teletón de Santiago.

Recibido: 25-10-2018 Aceptado: 24-05-2019

Correspondencia a: Andrea Solervicens $\mathrm{P}$. andreasolervicens@gmail.com

\section{ABSTRACT \\ Demographic and clinical-functional profile of patients with spinal muscular atrophy attending Instituto Teletón Santiago}

Introduction: Spinal muscular atrophy (SMA) is a severe neuromuscular disease (NMD) characterized by the loss of alpha motor neurons of the anterior horn of the spinal cord, causing progressive weakness of proximal muscles. Global prevalence is estimated to be $1 / 6,000-10,000$ live births. There is limited data on SMA patients in the Chilean population. Objective: To establish the scale, demographics and functional-clinical characteristics of SMA patients attending Instituto Teletón Santiago (ITS). Patients and Method: Cross-sectional, descriptive study based on the review of ITS's data information system, clinical records and telephone surveys. Results: 62 SMA patients were identified attending regular controls at ITS, $49(79 \%)$ were included in the study. $30.6 \%$, $36.7 \%$ and $32.7 \%$ correspond to SMA 1,2 or 3 , respectively. Average age 10.6 \pm 6.6 years; loss of gait at a median age of 6.8 years in SMA 3 patients. $67.3 \%$ require some type of breathing assistance, $44.9 \%$ have swallowing problems, $75.5 \%$ scoliosis, $49 \%$ hip dislocation or subluxation, $79.6 \%$ contracture of joints and $65.3 \%$ pain. In terms of social participation, $83 \%$ of recreational activities are carried out indoors, mainly related to the use of technology; and $77.5 \%$ go out to take part in social activities. Conclusion: Functional-clinical characteristics of SMA patients show that there is a group with high levels of dependence in all types of the disease, multiple concurrent disorders and secondary musculoskeletal conditions, and therefore, in the necessity to have a multidisciplinary rehabilitation system, as described in international literature.

Key words: Spinal muscular atrophy, demographics, functionality, neuromuscular disease.

\section{RESUMEN}

Introducción: La atrofia muscular espinal (AME) es una enfermedad neuromuscular (ENM) severa caracterizada por la degeneración de las motoneuronas alfa del asta anterior de la médula espinal, generando una debilidad progresiva de la musculatura proximal. La incidencia mundial se estima en 1/6.000-10.000 nacidos vivos. Contamos con escasos datos de las características de los pa- 
cientes con AME en la población chilena. Objetivo: Determinar magnitud, características demográficas y clínico funcionales de los pacientes con AME atendidos en el Instituto Teletón Santiago (ITS). Pacientes y Método: Estudio descriptivo transversal, basado en revisión del sistema informático ITS, fichas clínicas y encuesta telefónica. Resultados: Se identificaron 62 pacientes con AME con controles en ITS, 49 (79\%), fueron incluidos. 30,6\%, 36,7\% y 32,7\% corresponden a pacientes con AME 1, 2 y 3 respectivamente. Edad promedio $10,6 \pm 6,6$ años; pérdida de marcha edad mediana de 6,8 años en pacientes AME 3. 67,3\% requieren algún tipo de asistencia ventilatoria, $44,9 \%$ presentan trastornos de deglución, 75,5\% escoliosis, $49,0 \%$ subluxación o luxación de cadera, $79,6 \%$ retracciones articulares y $65,3 \%$ dolor. En participación, el $83 \%$ de las actividades de ocio se realizan dentro del hogar, principalmente de tipo tecnológico; 77,5\% realiza salidas sociales. Conclusión: Las características clínico funcionales de los pacientes con AME, evidencian un grupo que presenta un alto nivel de dependencia en todos los tipos de la enfermedad, múltiples comorbilidades y alteraciones músculo-esqueléticas secundarias y por ello requiere de un programa de rehabilitación multidisciplinaria tal como lo describe la literatura internacional.

Palabras clave: Atrofia muscular espinal, perfil demográfico, funcionalidad, enfermedad neuromuscular.

\section{Introducción}

La atrofia muscular espinal (AME) es un trastorno neurodegenerativo, autosómico recesivo, que se caracteriza por la degeneración de las neuronas motoras del asta anterior de la médula espinal ${ }^{1,2}$. Es causada por la ausencia o mutación puntual en el exón 7 del gen survival motor neuron 1 (SMN 1) ubicado en el cromosoma 5 (5q11.2-q13.3). La sobrevivencia depende de un gen homólogo, SMN 2, donde a modo general, cuantas más copias de éste, menor la gravedad clínica ${ }^{3-5}$.

Después de la fibrosis quística, la AME es la segunda causa de mortalidad infantil hereditaria $^{6,7}$. Su incidencia se estima entre 1 en 6.000 a 10.000 nacidos vivos ${ }^{6,3,8}$.

El cuadro clínico se caracteriza por paresia e hipotonía simétrica de predominio proximal y progresiva, que genera alteraciones en la ventilación, deglución y deformidades músculo-esqueléticas, entre otros. Se clasifica en 4 grupos según la clínica ${ }^{5,7,3}$. AME tipo 1 (Werdnig-Hoffmann) se inicia en los primeros 6 meses de vida, es el tipo más severo; los niños nunca logran sedente y su esperanza de vida es menor de dos años ${ }^{9}$; sin embargo, con los avances recientes en los cuidados de estos pacientes, ha aumentado significativamente su sobrevida ${ }^{9-11}$. En AME tipo 2 los síntomas aparecen entre los 7 y 18 meses de vida, logran sedente estable pero no marcha, su expectativa de vida llega a la edad adulta (52\% a los 40 años) ${ }^{1,3}$. AME tipo 3 (Kugelberg-Welander) inicia después de los 18 meses de vida, logran marcha en algún momento, su expectativa de vida no difiere significativamente de la población sana ${ }^{12,13}$; ésta se subclasifica en 3 a y 3 b. AME 3 a son aquellos que inician la clínica antes de los 3 años, donde solo el $22 \%$ de este grupo mantendrá la marcha a los 40 años y AME $3 b$ aquellos que inician la clínica después de los 3 años manteniendo la marcha a los 40 años en un 58,7\%. AME tipo 4 parte con debilidad muscular en la segunda o tercera década de la vida, su compromiso motor es leve y no presentan compromiso respiratorio o gastrointestinal ${ }^{9}$

La AME es una patología compleja, rara y de difícil manejo. En Chile se cuenta con escasa información sobre sus características demográficas, clínicas y funcionales. Este 
conocimiento es pilar fundamental para poder otorgar a los afectados respuesta a sus necesidades, ya que su pronóstico de morbimortalidad a corto y mediano plazo depende de la precocidad de la implementación de un tratamiento integral. Dentro de las acciones se encuentran: la incorporación temprana de asistencia ventilatoria no invasiva, que favorece el crecimiento costal y el volumen torácico; el uso de técnicas de asistencia de la tos desde edades tempranas, ya que se considera que las alteraciones en la capacidad de toser son la primera causa de morbilidad en los pacientes con enfermedad neuromuscular (ENM) $)^{14,15}$; proporcionar una vía de alimentación segura, manejo quirúrgico de la escoliosis en los casos que se produzca dolor o compromiso respiratorio9. Así el manejo de estos pacientes genera un gran desafío para los profesionales y centros de atención ${ }^{16}$.

En el Instituto Teletón Santiago (ITS) los pacientes con AME reciben tratamiento integral, con especial énfasis en mantener y mejorar la funcionalidad y prevenir, reducir y tratar las complicaciones ortopédicas asociadas.

Se plantea como objetivo, determinar la prevalencia de la enfermedad y las características demográficas y clínico funcionales de los pacientes con AME atendidos en el ITS.

\section{Pacientes y Método}

Estudio descriptivo, transversal. Se incluyó a todos los pacientes con diagnóstico de AME atendidos en el ITS a mayo de 2016 con estado activo en el sistema computacional, y con consentimiento informado. Se excluyó aquellos institucionalizados, que no respondieran el teléfono en tres oportunidades en días distintos o que no contaban con estudio genético. Proyecto aprobado por el Comité Ético Científico de la Sociedad Pro Ayuda del Niño Lisiado (Certificado $n^{\circ} 76 / 2016$ ).

La información se obtuvo mediante búsqueda en la base de datos de ITS disponible en el sistema informático y también fue recolectada mediante encuesta telefónica (validada por expertos) y revisión de fichas clínicas. Desde el sistema informático se recolectaron las siguientes variables: fecha de nacimiento, género y nivel socioeconómico, dato entregado por la familia del paciente en una entrevista al servicio social. De la encuesta telefónica se obtuvo: lugar de residencia, educación, control motor, edad al momento de perder la marcha cuando corresponda, capacidad de desplazarse en 5, 50 y 500 m (Functional Mobility Scale), uso de asistencia ventilatoria, controles con médico broncopulmonar y entrenamiento respiratorio, vía de alimentación, dificultades para alimentación por boca, actividades de la vida diaria (lavado de manos, bañarse, vestirse, cortar comida, transferencia), participación (amigos, salidas sociales y actividades de ocio), presencia de dolor y sus características. De las fichas clínicas se obtuvo: tipo de AME, antecedentes familiares de AME, exámenes realizados para el diagnóstico, uso de órtesis, mobiliario, complicaciones músculo-esqueléticas en cadera y/o columna y retracciones articulares.

Para el análisis estadístico se utilizó el software estadístico SPSS versión 17.0. Se calcularon medidas de resumen y de dispersión en las variables correspondientes.

\section{Resultados}

Se encontró 62 pacientes con diagnóstico de AME en el sistema informático. 13 pacientes fueron excluidos, 5 porque no fue posible contactarlos para encuesta telefónica y 8 que no contaban con la confirmación genética. Finalmente, 49 pacientes fueron incluidos, lo que corresponde al $0,53 \%$ de todos los pacientes con estado activo atendidos en el ITS a mayo de 2016.

La distribución según tipo de AME se detalla en Tabla 1, habiéndose encontrado los tipo 1, 2, y 3; de estos últimos 14 (87,5\%) corresponden al subtipo AME 3 a y $2(12,5 \%)$ a AME $3 b$.

La edad promedio fue de 10,6 $\pm 6,6$ años (rango 1-26,2), con un predominio del sexo masculino $(61,2 \%)$. E1 87,7\% residía en Santiago y el $61,2 \%$ pertenecía a nivel socioeconómico de extrema pobreza o nivel medio bajo.

En relación a la educación formal, 3 (20\%) 
Tabla 1. Características sociodemográficas

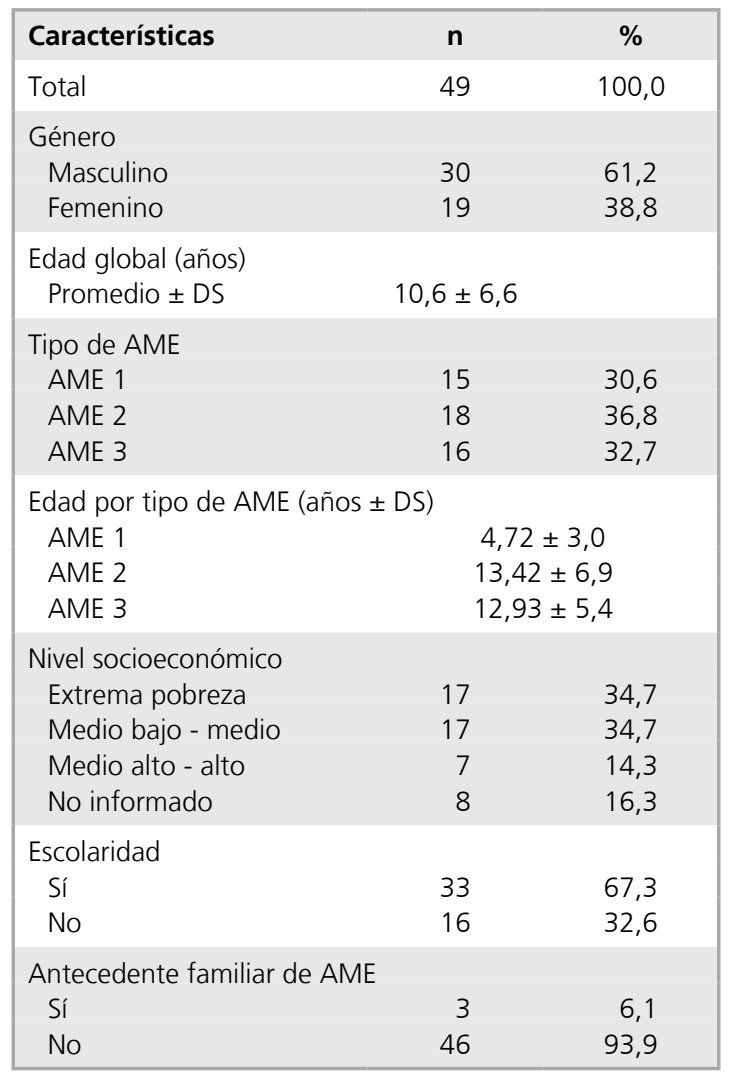

pacientes AME 1 recibían educación en su domicilio, 90\% de los pacientes con AME 2 y 3 recibía educación en algún centro educacional; de los pacientes que recibía algún tipo de educación $63,6 \%$ se encontraba cursando su etapa escolar y el 39,4\% participaba en el programa de integración escolar (PIE).

\section{Características clínico funcionales}

Control cefálico presentaba el $13,3 \%$ de los AME 1 y el 100,0\% de los AME 2 y 3 . Un sedente independiente se apreciaba en $0 \%$ de los pacientes AME 1, 44,4\% de los AME 2 y un $75,0 \%$ de los AME 3 .

La capacidad de marcha estaba presente en el 50,0\% de los pacientes AME 3, 42,8\% subtipo 3 a y $100,0 \%$ de los pacientes subtipo $3 \mathrm{~b}$. Los pacientes con AME 3 que perdieron la marcha presentaron una mediana de 6,8 años para dicho evento.

Un paciente con AME 1 y $8(44,0 \%)$ pacientes con AME 2 lograban desplazarse de forma independiente en $500 \mathrm{~m}$ utilizando silla de ruedas eléctrica; de los pacientes con AME 3 que lograban desplazamientos con marcha independiente un $61,6 \%$ lo hacía en $5 \mathrm{~m}$, un $58,4 \%$ en $50 \mathrm{~m}$ y un $40,0 \%$ en $500 \mathrm{~m}$ (Tabla 2 ).

Tabla 2. Desplazamiento independiente*

\begin{tabular}{|c|c|c|c|c|c|c|c|c|}
\hline \multirow[t]{2}{*}{ Desplazamiento logrado } & \multicolumn{2}{|c|}{ AME 1} & \multicolumn{2}{|c|}{ AME 2} & \multicolumn{2}{|c|}{ AME 3} & \multicolumn{2}{|c|}{ Total } \\
\hline & $\mathbf{n}$ & $\%$ & $\mathbf{n}$ & $\%$ & $\mathbf{n}$ & $\%$ & $\mathbf{n}$ & $\%$ \\
\hline Total & 15 & 100,0 & 18 & 100,0 & 16 & 100,0 & 49 & 100,0 \\
\hline $5 \mathrm{~m}$ & 1 & 6,7 & 13 & 72,2 & 13 & 81,3 & 27 & 55,1 \\
\hline Sin ayudas técnicas & - & - & - & & 6 & 46,2 & 6 & 22,2 \\
\hline Con bastones o carro & & - & - & & 2 & 15,4 & 2 & 7,4 \\
\hline Silla ruedas estándar & - & - & 4 & 30,8 & 4 & 30,8 & 8 & 29,6 \\
\hline Silla ruedas eléctrica & 1 & 100,0 & 9 & 69,2 & 1 & 7,7 & 11 & 40,7 \\
\hline $50 \mathrm{~m}$ & 1 & 6,7 & 10 & 55,6 & 12 & 75,0 & 23 & 46,9 \\
\hline Sin ayudas técnicas & - & - & - & & 5 & 41,7 & 5 & 21,7 \\
\hline Con bastones o carro & - & - & - & & 2 & 16,7 & 2 & 8,7 \\
\hline Silla ruedas estándar & - & - & 1 & 10,0 & - & - & 1 & 4,3 \\
\hline Silla ruedas eléctrica & 1 & 100,0 & 9 & 90,0 & 4 & 33,3 & 14 & 60,9 \\
\hline Gateo & - & - & - & & 1 & 8,3 & 1 & 4,3 \\
\hline $500 \mathrm{~m}$ & 1 & 6,7 & 8 & 44,4 & 10 & 62,5 & 19 & 38,8 \\
\hline Sin ayudas técnicas & - & - & - & - & 3 & 30,0 & 3 & 15,8 \\
\hline Con bastones o carro & - & - & - & - & 1 & 10,0 & 1 & 5,3 \\
\hline Silla ruedas estándar & - & - & - & - & 2 & 20,0 & 2 & 10,5 \\
\hline Silla ruedas eléctrica & 1 & 100,0 & 8 & 100,0 & 4 & 40,0 & 13 & 68,4 \\
\hline
\end{tabular}

*Modo de desplazamiento calculado en base al n total que lo logra. 
Del total el 67,3\% requirió asistencia ventilatoria (AV), ya sea ventilación mecánica invasiva (VMI) o no invasiva (BiPAP). Todos los pacientes con AME 1 contaban con apoyo ventilatorio, de ellos el $86,7 \%$ estaba con VMI de uso continuo. El $72,2 \%$ de los AME 2 y el $31,3 \%$ de los pacientes AME 3, utilizó BiPAP.

E1 81,6\% refirió tener controles regulares con médico broncopulmonar y un $63,3 \%$ dijo realizar algún tipo de entrenamiento de la musculatura respiratoria (válvula, ambu o técnicas de asistencia de la tos).

La dificultad para alimentación por vía oral se presentó en el 100,0\% de los AME 1, el 38,9\% de los AME 2 y ningún paciente con AME 3 (Tabla 3).

En relación al grado de independencia en las actividades de la vida diaria (AVD), el $100,0 \%$ de los pacientes AME 1 requirió asistencia parcial o total para bañarse, vestirse, cortar alimento y transferencias. El 38,9\% de los pacientes AME 2 logró realizar aseo menor en forma independiente y el $100,0 \%$ requirió asistencia en el resto de las AVD evaluadas. E1 85,7\% de los pacientes AME 3 necesitó algún tipo de asistencia para bañarse, $57,1 \%$ para vestirse y el $62,5 \%$ para realizar las transferencias (Tabla 4).

\section{Participación y actividades de ocio}

En participación, un 69,3\% reportó tener amigos y un $77,5 \%$ realizar salidas sociales. En actividades de ocio, se observó un 83,0\% de actividades dentro del hogar donde las actividades con equipos electrónicos, TV, computador, juegos de consola abarcan el $43,0 \%$ de éstas.

\section{Dolor}

Al momento de realizar la encuesta telefónica, un $65,3 \%$ refirió presencia de dolor en los últimos 30 días con una frecuencia mayor en AME 2 y AME 3, 77,8\% y 75,0\% respectivamente. En los pacientes AME 1 el dolor fue reportado en un 40,0\% $(p<0,046)$. E1 50,0\% de los pacientes presentó dolor de intensidad moderada (EVA 4-6), y de ellos el $46,9 \%$ recibe tratamiento. En cuanto al origen del dolor un $85,7 \%$ impresionó de origen músculo-esquelético en una o más localizaciones; el 40,6\% refirió dolor de espalda, 34,3\% en extremidades inferiores, $28,1 \%$ en caderapelvis y $15,6 \%$ en extremidades superiores.

\section{Órtesis y mobiliario}

$78,9 \%$ de los pacientes utilizó órtesis (palmetas, órtesis tobillo pie, canaletas, inserto

Tabla 3. Manejo respiratorio y vía de alimentación

\begin{tabular}{|c|c|c|c|c|c|c|c|c|}
\hline \multirow[t]{2}{*}{ Variables } & \multicolumn{2}{|c|}{ AME 1} & \multicolumn{2}{|c|}{ AME 2} & \multicolumn{2}{|c|}{ AME 3} & \multicolumn{2}{|c|}{ Total } \\
\hline & $\mathbf{n}$ & $\%$ & $\mathbf{n}$ & $\%$ & $\mathbf{n}$ & $\%$ & $\mathbf{n}$ & $\%$ \\
\hline Total & 15 & 100,0 & 18 & 100,0 & 16 & 100,0 & 49 & 100,0 \\
\hline Asistencia ventilatoria (AV) & 15 & 100,0 & 13 & 72,2 & 5 & 31,3 & 33 & 67,3 \\
\hline $\begin{array}{l}\text { Tipo AV } \\
\text { BiPAP } \\
\text { VMI }\end{array}$ & $\begin{array}{r}2 \\
13\end{array}$ & $\begin{array}{l}13,3 \\
86,7\end{array}$ & $\begin{array}{c}13 \\
-\end{array}$ & $\begin{array}{c}100,0 \\
-\end{array}$ & $\begin{array}{l}5 \\
-\end{array}$ & $\begin{array}{c}100,0 \\
-\end{array}$ & $\begin{array}{l}20 \\
13\end{array}$ & $\begin{array}{l}60,6 \\
39,4\end{array}$ \\
\hline $\begin{array}{l}\text { Tiempo de uso AV } \\
\text { Tiempo parcial } \\
\text { Uso continuo }\end{array}$ & $\begin{array}{r}2 \\
13\end{array}$ & $\begin{array}{l}13,3 \\
86,7\end{array}$ & $\begin{array}{r}11 \\
2\end{array}$ & $\begin{array}{l}84,6 \\
15,4\end{array}$ & $\begin{array}{l}5 \\
-\end{array}$ & $\begin{array}{c}100,0 \\
-\end{array}$ & $\begin{array}{l}18 \\
15\end{array}$ & $\begin{array}{l}54,5 \\
45,5\end{array}$ \\
\hline Controles broncopulmonar & 12 & 80,0 & 18 & 100,0 & 10 & 62,5 & 40 & 81,6 \\
\hline Entrenamiento respiratorio & 9 & 60,0 & 12 & 66,7 & 10 & 62,5 & 31 & 63,3 \\
\hline $\begin{array}{l}\text { Vía de alimentación } \\
\text { Vía oral con signos de aspiración } \\
\text { Sonda nasogástrica } \\
\text { Gastrostomía }\end{array}$ & $\begin{array}{r}1 \\
14\end{array}$ & $\begin{array}{r}- \\
6,7 \\
93,3\end{array}$ & $\begin{array}{c}6 \\
- \\
1\end{array}$ & $\begin{array}{c}33,3 \\
- \\
5,5\end{array}$ & $\begin{array}{l}- \\
-\end{array}$ & $\begin{array}{l}- \\
-\end{array}$ & $\begin{array}{r}6 \\
1 \\
15\end{array}$ & $\begin{array}{r}33,3 \\
2,0 \\
30,6\end{array}$ \\
\hline
\end{tabular}

Tipo y tiempo de AV calculado sobre el $\mathrm{n}$ total de aquellos que requieren la asistencia. 
Tabla 4 Independencia en actividades de la vida diaria

\begin{tabular}{|c|c|c|c|c|c|c|c|c|}
\hline \multirow[t]{2}{*}{ Independencia en AVD } & \multicolumn{2}{|c|}{ AME 1} & \multicolumn{2}{|c|}{ AME 2} & \multicolumn{2}{|c|}{ AME 3} & \multicolumn{2}{|c|}{ Total } \\
\hline & $\mathbf{n}$ & $\%$ & $\mathbf{n}$ & $\%$ & $\mathbf{n}$ & $\%$ & $\mathbf{n}$ & $\%$ \\
\hline Lavado manos > 3 años $(n=44)$ & 10 & & 18 & & 16 & & 44 & \\
\hline Independiente & 1 & 10,0 & 7 & 38,9 & 14 & 87,5 & 22 & 50,0 \\
\hline Dependiente* & 9 & 90,0 & 11 & 61,1 & 2 & 12,5 & 22 & 50,0 \\
\hline Bañarse $>5$ años $(n=37)$ & 6 & & 17 & & 14 & & 37 & \\
\hline Independiente & - & - & - & - & 2 & 14,3 & 2 & 5,4 \\
\hline Dependiente* & 6 & 100,0 & 17 & 100,0 & 12 & 85,7 & 35 & 94,6 \\
\hline Vestirse $>5$ años $(n=37)$ & 6 & & 17 & & 14 & & 37 & \\
\hline Independiente & - & - & - & - & 6 & 42,9 & 6 & 16,2 \\
\hline Dependiente* & 6 & 100,0 & 17 & 100,0 & 8 & 57,1 & 31 & 83,8 \\
\hline Cortar comida > 5 años $(n=37)$ & 6 & & 17 & & 14 & & 37 & \\
\hline Independiente & - & - & - & - & 7 & 50,0 & 7 & 18,9 \\
\hline Dependiente* & 6 & 100,0 & 17 & 100,0 & 7 & 50,0 & 30 & 81,1 \\
\hline Transferencia $>2$ años $(n=47)$ & 13 & & 18 & & 16 & & 47 & \\
\hline Independiente & - & - & - & - & 6 & 37,5 & 6 & 12,8 \\
\hline Dependiente* & 13 & 100,0 & 18 & 100,0 & 10 & 62,5 & 41 & 87,2 \\
\hline
\end{tabular}

*Dependiente: parcial o completo. Para cada hito motor se ajustó la población según edad de adquisición de dicho hito.

plantar y/o corsé), con similar frecuencia en los distintos tipos de AME. E1 55,1\% de los pacientes utilizó algún tipo de seating y/o bipedestador. De los pacientes AME 1 el 93,3\% usó seating y 40,0\% bipedestador; $44,4 \%$ de los pacientes AME 2 utilizó bipedestador y el mismo porcentaje utilizó seating. El 6,3\% de los pacientes AME 3 usó algún mobiliario.

\section{Deformidades músculo-esqueléticas}

La presencia de escoliosis se observó en 37 pacientes $(75,5 \%)$ y 10 de éstos $(27,0 \%)$ fueron sometidos a cirugía. La subluxación o luxación de cadera se reportó en 24 casos (49,0\%). Las contracturas articulares se presentaron en un $36,7 \%$ en extremidades superiores y $79,6 \%$ en extremidad inferiores. En todos los tipos AME se presentaron retracciones en proporciones relevantes, especialmente en tobillo-pie, rodillas, caderas y codos, fluctuando entre el 40,0\% y el 93,3\% (Tabla 5).

\section{Discusión}

Si bien la población de pacientes con diagnóstico de AME resulta pequeña en comparación con toda la población de pacientes atendidos en ITS $(0,53 \%)$, constituye un grupo con elevados requerimientos, dados por el gran nivel de dependencia que experimentan los pacientes y por la alta morbimortalidad asociada al diagnóstico.

En nuestro estudio, AME 1 presenta una frecuencia menor a lo reportado por la literatura internacional ${ }^{1}$ que es de un $50,0 \%{ }^{6}$; esto podría deberse a que Teletón es un centro de rehabilitación ambulatorio, al que muchos pacientes podrían no alcanzar a ser derivados debido a su mayor severidad y alta tasa de mortalidad dentro de los primeros 12 meses de vida. Sin embargo con respecto a su nivel de dependencia, requerimiento de asistencia ventilatoria y comorbilidades músculo-esqueléticas se comportan de manera similar a lo reportado en la literatura ${ }^{1,11,19,26,27}$.

Se aprecia que el grado de dependencia es proporcional a la severidad de la enfermedad, mayor en los pacientes con AME 1 y menor en aquellos con AME 3.

Nos parece especialmente interesante el análisis de las características funcionales; dentro de ellas, la capacidad para desplazarse de manera independiente, donde a la luz de los resultados, parece indispensable que los pacientes cuenten con silla de ruedas eléctricas 
Tabla 5 Deformidades músculo-esqueléticas

\begin{tabular}{|c|c|c|c|c|c|c|c|c|}
\hline \multirow[t]{2}{*}{ Deformidades músculo-esqueléticas } & \multicolumn{2}{|c|}{ AME 1} & \multicolumn{2}{|c|}{ AME 2} & \multicolumn{2}{|c|}{ AME 3} & \multicolumn{2}{|c|}{ Total } \\
\hline & $\mathbf{n}$ & $\%$ & $\mathbf{n}$ & $\%$ & $\mathbf{n}$ & $\%$ & $\mathbf{n}$ & $\%$ \\
\hline Total & 15 & 100,0 & 18 & 100,0 & 16 & 100,0 & 49 & 100,0 \\
\hline \multicolumn{9}{|l|}{ Escoliosis } \\
\hline Sí & 7 & 46,7 & 18 & 100,0 & 12 & 75,0 & 37 & 75,5 \\
\hline No & 4 & 26,7 & - & & 2 & 12,5 & 6 & 12,2 \\
\hline No informado & 4 & 26,7 & - & & 2 & 12,5 & 6 & 12,2 \\
\hline Cirugía escoliosis & - & - & 5 & 27,7 & 5 & 31,2 & 10 & 27,0 \\
\hline \multicolumn{9}{|l|}{ Sub/luxación cadera } \\
\hline Sí & 7 & 46,7 & 13 & 72,2 & 4 & 25,0 & 24 & 49,0 \\
\hline No & 1 & 6,7 & - & & 1 & 6,3 & 2 & 4,1 \\
\hline No informado & 7 & 46,7 & 5 & 27,8 & 11 & 68,8 & 23 & 46,9 \\
\hline Cirugía sub/luxación & - & & - & & 1 & 6,3 & 1 & 4,1 \\
\hline Retracción articular EESS* & - & & & & & & & \\
\hline Sí & 4 & 26,7 & 10 & 55,6 & 4 & 25,0 & 18 & 36,7 \\
\hline No & 11 & 73,3 & 8 & 44,4 & 12 & 75,0 & 31 & 63,3 \\
\hline \multicolumn{9}{|l|}{ Retracción articular EEII* } \\
\hline Sí & 13 & 86,7 & 16 & 88,9 & 10 & 62,5 & 39 & 79,6 \\
\hline No & 2 & 13,3 & 2 & 11,1 & 6 & 37,5 & 10 & 20,4 \\
\hline \multicolumn{9}{|l|}{ Ubicación de la retracción } \\
\hline Tobillo-pie & 14 & 93,3 & 16 & 88,9 & 11 & 68,8 & 41 & 83,7 \\
\hline Rodilla & 9 & 60,0 & 16 & 88,9 & 7 & 43,8 & 32 & 65,3 \\
\hline Cadera & 6 & 40,0 & 11 & 61,1 & 5 & 31,3 & 22 & 44,9 \\
\hline Muñeca-mano & 2 & 13,3 & 5 & 27,8 & 2 & 12,5 & 9 & 18,4 \\
\hline Hombro & 2 & 13,3 & 2 & 11,1 & 1 & 6,3 & 5 & 10,2 \\
\hline Codo & 4 & 26,7 & 8 & 44,4 & 2 & 12,5 & 14 & 28,6 \\
\hline
\end{tabular}

* EESS: extremidades superiores. EEll: extremidades inferiores. Sub/luxación: subluxación o luxación.

para facilitar su participación tanto en espacios intradomiciliarios como comunitarios. En relación a este punto, Dunaway ${ }^{18}$ propone que los niños con AME tipo 2 deberían usar una SR eléctrica desde los dos años de vida; considerando lo relevante de fomentar la participación, también creemos necesario optimizar el acceso a SR eléctrica de manera precoz. La capacidad para desplazarse con marcha independiente varía según el tipo y subtipo de AME; en nuestro estudio encontramos que un $42,8 \%$ de los AME 3 a y todos los $3 \mathrm{~b}$ mantenían marcha independiente, lo que, considerando la historia natural de la enfermedad, es compatible con la información reportada por Zerres ${ }^{17}$ en pacientes de 40 años de edad donde un 22,0\% de los AME 3a y un $58,7 \%$ de los AME $3 \mathrm{~b}$ mantienen la marcha.

La participación fue evaluada por la presencia de amigos, salidas sociales y actividades de ocio, donde se refleja que su compromiso motor les dificulta la participación en sociedad, prefiriendo actividades dentro del hogar y de tipo tecnológico. En este sentido, el mundo actual y el avance de la tecnología y la comunicación mediante redes, se nos presentan como una posibilidad de rehabilitación y de acceso a oportunidades para nuestros pacientes.

Existen escasos reportes en la literatura en cuanto al nivel de independencia y de AVD que pueden desarrollar los pacientes con AME, por lo que quisimos explorar de manera gruesa algunas AVD y algunos ítems de participación social utilizando la Clasificación Internacional del Funcionamiento, de la Discapacidad y de la Salud (CIF). Febrer ${ }^{19}$, por ejemplo, señala que los niños con AME no suelen alcanzar el nivel de independencia que les correspondería por la edad, lo que es compatible con lo que encontramos en nuestro estudio, y por lo demás, 
predecible dado el nivel de compromiso motor que presentan los pacientes. Considerando todas estas limitaciones en AVD, estamos de acuerdo con $\mathrm{Fadol}^{20}$, en la importancia de disminuir las barreras mediante tecnología asistida, productos de soporte u otros, para fomentar la independencia y mejorar su calidad de vida.

Nos parece interesante destacar el aporte que significa explorar las AVD de manera específica para poder conocer mejor a nuestra población y orientar los entrenamientos en AVD a sus necesidades reales. Por ejemplo, en el caso de los pacientes AME 1 poner el foco en el entrenamiento del cuidador, en los pacientes AME 2 potenciar la independencia en AVD de aseo menor y alimentación y en los pacientes AME 3 poner un mayor énfasis en AVD más exigentes acordes a sus posibilidades, por ejemplo, facilitar el aseo mayor con las adaptaciones, infraestructura y entrenamiento que ello requiera.

La principal causa de morbimortalidad en $\mathrm{AME}^{21}$ es respiratoria, por lo que el Consensus Statement for Standard of Care in Spinal Muscular Atrophy $2007^{9}$ sugiere un control respiratorio cada 3 a 6 meses. En nuestro estudio no todos los pacientes refirieron controles regulares con médico broncopulmonar y casi un $40,0 \%$ comunicó no realizar entrenamiento respiratorio (tos asistida manual o mecánica y entrenamiento con válvulas threshold). Esto puede obedecer a varios motivos; a una falta de claridad de los pacientes de cuáles son sus tratantes, al sesgo de memoria al que está sujeta la encuesta o a la falta real de control con médico broncopulmonar. Por lo tanto, estos datos nos ponen en alerta y nos obligan a reforzar la educación, la información completa a los padres y las derivaciones al nivel hospitalario para mejorar esta situación. En ITS contamos con atenciones de rehabilitación respiratoria para los pacientes con enfermedades neuromusculares; dado su relevancia, sería deseable un mayor desarrollo de esta área, unificar protocolos en todos los Institutos Teletón del país como también reforzar la red de derivación con el nivel hospitalario.

La contrarreferencia asistida es prioritaria, la información completa a los padres y el ejercicio de toma de decisiones bioéticas es fundamental, más aún, en un momento del desarrollo científico donde los padres están buscando activamente los tratamientos curativos de base genética.

La literatura propone el uso de asistencia ventilatoria no invasiva (AVNI), incluso para pacientes AME $1^{1}$, sin embargo, nosotros encontramos que casi la totalidad de los pacientes AME 1 atendidos en ITS se encuentra con ventilación invasiva. Esta situación dista de lo reportado por Bladen ${ }^{1}$ que describe en su estudio con 5.000 pacientes, que el 57,0\% de los pacientes con AVNI corresponden a AME 1. Esta discrepancia nos hace reflexionar respecto del rol educador y orientador que puede tener el equipo de rehabilitación en torno a este tema.

Otro punto interesante de discutir es el hallazgo en torno al dolor, pues encontramos que, un $65,3 \%$ de los pacientes refirió presencia de dolor el mes previo a la encuesta. Según Miró $^{22}$, la mayoría de los pacientes con ENM de progresión lenta presentan dolor crónico en alguna medida. Otro elemento relevante es que menos de la mitad de ellos se encontraban con tratamiento específico; esto, tal como plantea López ${ }^{23}$, probablemente obedece al subdiagnóstico del dolor en las patologías crónicas infantiles. Por ello proponemos buscar en forma dirigida y sistemática la presencia de dolor y en el caso de ser detectado, tomar una conducta más activa en su manejo y seguimiento.

De las complicaciones ortopédicas, la frecuencia de escoliosis y de su manejo quirúrgico son concordantes con la bibliografía internacional, donde los pacientes AME 2 presentan la mayor frecuencia afectando al $100 \%{ }^{24-27}$. Por otra parte, destaca un alto porcentaje de contracturas articulares, principalmente de extremidades inferiores, evidenciando la necesidad de un manejo precoz para prevenirlas y así disminuir el dolor y las limitaciones de las AVD que éstas generan.

Las limitaciones encontradas en este estudio son el sesgo de memoria en la entrevista telefónica y el registro en ficha clínica, que no cuenta con un formato estándar, por lo que en ocasiones no fue posible encontrar toda la información. 
Lamentamos que 8 pacientes fueran excluidos del estudio por no contar con estudio genético; estos pacientes fueron contactados y se les ofreció realizar el examen.

Consideramos fundamental utilizar escalas de medición validadas para pacientes con ENM que permitan evaluar su condición y medir de manera más objetiva el resultado de nuestras intervenciones. Sugerimos el uso de Hammersmith Functional Motor Scale ${ }^{28}$ y el Revised Upper Limb Module ${ }^{29}$. Ambas escalas son fáciles y rápidas de aplicar y constituyen una buena herramienta para evaluar la funcionalidad de los pacientes con AME.

El valor agregado de este estudio es reportar la funcionalidad en términos de la CIF y esto pretende abrir la puerta para que futuras investigaciones estudien los déficits, las limitaciones de la actividad y las restricciones de la participación de manera más profunda.

\section{Agradecimientos}

Agradecemos a los pacientes y sus familias que aceptaron participar en este estudio, a los profesionales de la dirección de investigación y desarrollo (DIDE) de Teletón.

\section{Referencias bibliográficas}

1. Bladen CL, Thompson R, Jackson JM, Garland C, Wegel C, Ambrosini A, et al. Mapping the differences in care for 5,000 Spinal Muscular Atrophy patients, a survey of 24 national registries in North America, Australasia and Europe. J Neurol 2014; 261 (1): 152-63.

2. Nascimiento A, Tizzano E, Colomer J. Diagnóstico, conceptos genéticos y clasificación de la atrofia muscular espinal, neuropatías hereditarias y miastenias. En: Rehabilitación de las Enfermedades Neuromusculares en la Infancia. Barcelona-España: Panamericana; 2015. p. 17-32.

3. Kaufmann P, McDermott M, Darras B, Finkel R, Kang $\mathrm{P}$, Oskoui M, et al. Observational study of spinal muscular atrophy type 2 and 3 : functional outcomes over 1 year. Arch Neurol 2011; 68 (6): 779-86.

4. Cuisset J-M, Estournet B. Recommendations for the diagnosis and management of typical childhood spinal muscular atrophy. Rev Neurol 2012; 168 (12): 902-9.
5. Guillot N, Cuisset J-M, Cuvellier J-C, HurteventJ-F, Joriot S, Vallee L. Unusual clinical features in infantile Spinal Muscular Atrophies. Rev Brain Dev. 2008; 30 (3): 169-78.

6. D'Amico A, Mercuri E, Tiziano FD, Bertini E. Spinal muscular atrophy. Orphanet J Rare Dis 2011; 6 (1): 71.

7. Kaufmann P, McDermott MP, Darras BT, Finkel RS, Kang PB, Foley AR, et al. Prospective cohort study of spinal muscular atrophy types 2 and 3. Am Acad Neurol. 2012; 1889-97.

8. Kolb S, Kissel J. Spinal muscular atrophy: a timely review. Arch Neurol. 2011; 68 (8):979-84.

9. Wang C, Finkel R, Bertini E, Schroth M, Simonds A, Wong B, et al. Consensus Statement for Standard of Care in Spinal Muscular Atrophy. J Child Neurol 2007; 22 (8): 1027-49.

10. Oskoui M, Levy G, Garland C, Gray J, O’Hagen J, De Vivo DC, et al. The changing natural history of spinal muscular atrophy type 1. Neurol 2007; 69 (20): 1931-6.

11. Lunn MR, Wang CH. Seminar: Spinal muscular atrophy. Lancet 2008; 371: 2120-33.

12. Viollet L, Melki J. Spinal muscular atrophies. 1st ed. Handbook of Clinical Neurology. Elsevier B.V. 2013; 1395-411.

13. Markowitz JA, Singh P, Darras BT. Spinal Muscular Atrophy: A Clinical and Research Update. Pediatr Neurol. 2012; 46 (1): 1-12.

14. Servera E, Sancho J, Zafra M. Tos y enfermedades neuromusculares. Manejo no invasivo de las secreciones respiratorias. Arch Bronconeumol 2003; 39 (9): 418-27.

15. Benditt JO. Initiating noninvasive management of respiratory insufficiency in neuromuscular disease. Pediatrics 2009; 123 Suppl: S236-8.

16. Stavarachi M, Apostol P, Toma M, Cimponeriu D, Gavrila L. Spinal muscular atrophy disease: a literature review for therapeutic strategies. J Med Life 2010; 3 (1): 3-9.

17. Zerres K, Rudnik-Schöneborn S, Forrest E, Lusakowska A, Borkowska J, Hausmanowa-Petrusewicz I. A collaborative study on the natural history of childhood and juvenile onset proximal spinal muscular atrophy (type II and III SMA): 569 patients. J Neurol Sci 1997; 146 (1): 67-72.

18. Dunaway S, Montes J, O'Hagen J, Sproule DM, Vivo DC DE, Kaufmann P. Independent mobility after early introduction of a power wheelchair in spinal muscular atrophy. J Child Neurol 2013; 28 (5): 576-82.

19. Febrer A, Meléndez M. Atrofia muscular espinal. Complicaciones y rehabilitación. Rehabilitación 2001; 35 (5): 307-11. 
20. FadoL L, Febrer A. Tecnología asistida y ayuda a la dependencia. En: Rehabilitación de las Enfermedades Neuromusculares en la Infancia. Barcelona-España: panamericana; 2015. p. 125-35.

21. Cols M, del Campo E. Manejo de las complicaciones respiratorias. En: Rehabilitación de las Enfermedades Neuromusculares en la Infancia. Barcelona-España: panamericana; 2015. p. 99-110.

22. Miró J, Gertz K, Carter G, Jensen M. Chronic Pain in Neuromuscular Disease. Pain Site and Intensity Differentially Impacts Function. Phys Med Rehabil Clin N Am. 2012; 23 (4): 895-902.

23. López M, Miró J. Dolor en niños y adolescentes con enfermedades neuromusculares. Rev Soc Esp Dolor 2013; 20 (3): 142-9.

24. Carter GT, Abresch RT, Fowler WM, Johnson ER, Kilmer DD, McDonald CM. Profiles of neuromuscular diseases. Spinal muscular atrophy. Am J Phys Med
Rehabil 1995; S150-9.

25. Sucato DJ. Spine deformity in spinal muscular atrophy. J Bone Joint Surg Am 2007; 89 Suppl 1: 148-54.

26. Tsirikos A, Baker A. Spinal muscular atrophy: Classification, etiology, and treatment of spinal deformity in children and adolescents. Curr Orthop 2006; 20 (6): 430-45.

27. Mesfin A, Sponseller P, Leet A. Spinal Muscular Atrophy: Manifestations and Management. J Am Acad Orthop Surg 2012; 20 (6): 393-401.

28. Main M, Kairon H. The Hammersmith functional motor scale for children with spinal muscular atrophy: a scale to test ability and monitor progress in children with limited ambulation. Eur J Paediatr Neurol 2003; 7: 155-9.

29. Mazzone ES, Mayhew A. Revised upper limb module for spinal muscular atrophy: Development of a new module. Muscle Nerve 2017; 55 (6): 869-74. 